\title{
Numerical modelling of swirling diffusive flames
}

\author{
Teresa Parra-Santos ${ }^{1, a}$, Ruben Perez ${ }^{2}$, Robert Z. Szasz ${ }^{3}$, Artur N. Gutkowski ${ }^{4}$ and Francisco Castro ${ }^{1}$ \\ ${ }^{1}$ University of Valladolid, Department of Energy and Fluid Mechanics, Valladolid, Spain \\ ${ }^{2}$ Tecnicas Reunidas, Thermo-Hydraulic Section, Madrid, Spain \\ ${ }^{3}$ Lund University, Energy Division, Lund, Sweden \\ ${ }^{4}$ Technical University of Lodz, Department of Heat Technology and Refrigeration, Lodz, Poland
}

\begin{abstract}
Computational Fluid Dynamics has been used to study the mixing and combustion of two confined jets whose setup and operating conditions are those of the benchmark of Roback and Johnson. Numerical model solves 3D transient Navier Stokes for turbulent and reactive flows. Averaged velocity profiles using RNG swirl dominated $\mathrm{k}$-epsilon model have been validated with experimental measurements from other sources for the non reactive case. The combustion model is Probability Density Function. Bearing in mind the annular jet has swirl number over 0.5, a vortex breakdown appears in the axis of the burner. Besides, the sudden expansion with a ratio of 2 in diameter between nozzle exits and the test chamber produces the boundary layer separation with the corresponding torus shape recirculation. Contrasting the mixing and combustion models, the last one produces the reduction of the vortex breakdown.
\end{abstract}

\section{Introduction}

An analysis was carried out to study numerically the interaction of two coaxial jets with different compositions, temperatures and axial velocities.

The geometrical set up corresponds with the case of Roback and Johnson [1], see figure 1, being the diameter of the combustion chamber the double of the outer diameter of the annular nozzle. In addition, the annual nozzle has eight fixed blades, swirler, that produce azimuthal velocity, [2].

Swirl number is defined as the ratio between the azimuthal and the axial momentum. When Swirl number is over a critical value, the radial distribution of pressure promotes reverse flow near the longitudinal axis of the chamber. This inner recirculation zone (IRZ) is known as vortex breakdown.

An increase of the swirl number usually means an increase of the IRZ's size, [3]. That is the reason of using swirling flows to promote enhanced mixture.

This work is devoted to high swirl number to study its flow pattern and its effect on the diffusion flame.

\section{Numerical model}

The Reynolds number based on bulk velocity at the test chamber corresponds to a transitional turbulence regime.

Navier Stokes equations are solved in a 3D computational domain with about 600 thousand cells. Figures 2 show a detail of the mesh on the swirler and in a longitudinal slice.

\footnotetext{
${ }^{a}$ Corresponding author: tpsantos@mail.com
}

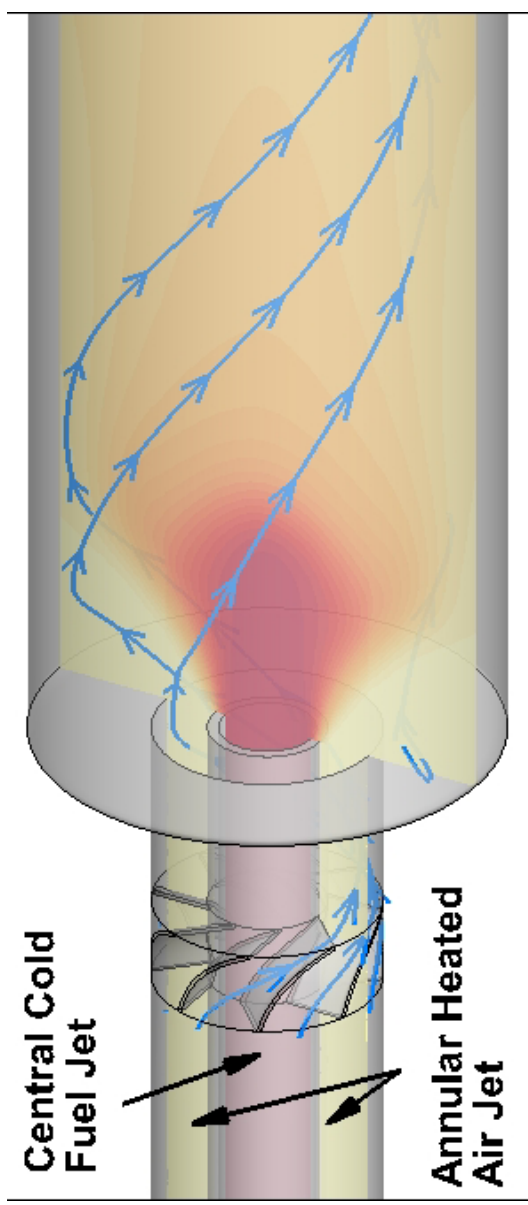

Fig. 1. Set up of the physical domain of Roback and Johnson burner 


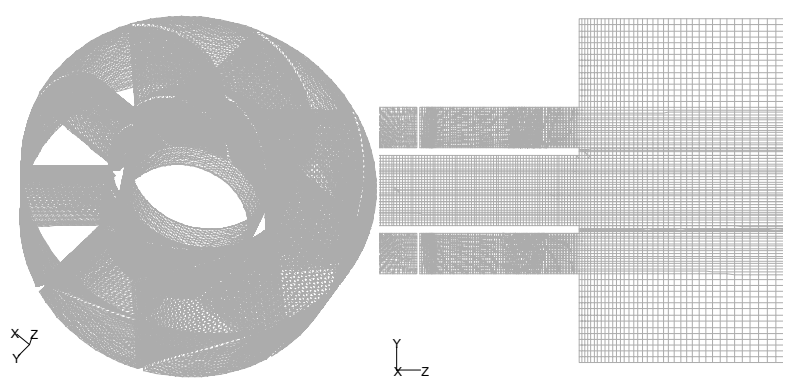

Fig. 2. Details of the mesh swirler (left) longitudinal slice (right).

The swirler has tetrahedral mesh whereas the nozzle and the chamber have a hexahedral mesh. As for the mesh quality, table 1 summarizes the statistics of equiangle skew, i.e. the dispersion of the hexahedral cell from angle $90^{\circ}$ and the tetrahedral cells from angle $60^{\circ}$. Also the aspect ratio is analysed.

Table 1. Mesh quality statistic.

\begin{tabular}{|c|c|c|c|c|}
\hline & & $\begin{array}{l}\text { Very } \\
\text { Good }\end{array}$ & Slightly Bad & Very Bad \\
\hline \multirow{2}{*}{$\begin{array}{l}\text { Equi- } \\
\text { Angle } \\
\text { skew }\end{array}$} & Range & $(0-0.3)$ & $(0.5,0.7)$ & $(0.7,1.0)$ \\
\hline & $\%$ cells & $88.86 \%$ & $1.88 \%$ & $0.84 \%$ \\
\hline \multirow{2}{*}{$\begin{array}{l}\text { Aspect } \\
\text { ratio }\end{array}$} & Range & $(1,5)$ & $(10,50)$ & $(50,200)$ \\
\hline & $\%$ cells & $73.96 \%$ & $16.57 \%$ & $0.42 \%$ \\
\hline
\end{tabular}

The numerical scheme is second order accuracy. SIMPLE is the method to coupling pressure and velocity resolution.

Two different simulations were conducted: nonreactive flow and reactive flow.

\subsection{Boundary conditions}

The boundary conditions and the geometrical details of the Roback and Johnson burner [1] are summarized in table 2. The nozzles discharge in the test chamber with a sudden expansion of 4 in area. The length of the chamber is $1 \mathrm{~m}$ but the refinement occurs in the first $300 \mathrm{~mm}$ where the flow patterns are complex.

Table 2. Summary of geometrical details and the inlet conditions for the nozzles.

\begin{tabular}{lcc}
\hline \multicolumn{1}{c}{ Magnitude } & Central jet & Annular inlet \\
\hline Diameter $(\mathrm{m})$ & $0-0.025$ & $0.0306-0.059$ \\
Velocity $\left(\mathrm{ms}^{-1}\right)$ & 0.66 & 1.52 \\
$\begin{array}{l}\text { Intensity of } \\
\text { turbulence }(\%)\end{array}$ & 12 & 7.5 \\
Temperature (K) & 300 & 1200 \\
Mass Fraction (-) & $0.234 \mathrm{CH}_{4}$ & $0.22 \mathrm{O}_{2}+0.78 \mathrm{~N}_{2}$ \\
Viscosity $\left(\mathrm{kg} \mathrm{m}^{-1} \mathrm{~s}^{-1}\right)$ & $1.08710^{-3}$ & $1.789410^{-5}$ \\
$\begin{array}{l}\text { Molecular Weight } \\
\left(\mathrm{kg} \mathrm{kmol}^{-1}\right)\end{array}$ & 16.043 & 28.966 \\
$\begin{array}{l}\text { Specific Heat } \\
\text { Transfer }\left(\mathrm{J} \mathrm{kg}^{-1} \mathrm{~K}^{-1}\right)\end{array}$ & Polynomial function of temperature \\
\hline
\end{tabular}

\subsection{Turbulence model}

The turbulence model was swirl-dominated RNG k- $\varepsilon$ model because it is specially recommended for large zones with relevant vorticity. A detailed comparison of different turbulence models is presented in [2].

Two additional conservation equations are solved. One is for the turbulent kinetic energy, eq. (1), and another for its dissipation, eq. (2). The influence of swirling is represented by the term $R_{\varepsilon}$ that depends on the strain tensor, [4].

$$
\begin{gathered}
\frac{\partial}{\partial t}(\rho k)+\frac{\partial}{\partial x_{i}}\left(\rho k u_{i}\right)=\frac{\partial}{\partial x_{j}}\left(\alpha_{k} \mu_{e f f} \frac{\partial k}{\partial x_{j}}\right)+G_{k}-\rho \varepsilon \\
\frac{\partial}{\partial t}(\rho \varepsilon)+\frac{\partial}{\partial x_{i}}\left(\rho \varepsilon u_{i}\right)=\frac{\partial}{\partial x_{j}}\left(\alpha_{\varepsilon} \mu_{e f f} \frac{\partial \varepsilon}{\partial x_{j}}\right)+ \\
+C_{1 \varepsilon} \frac{\varepsilon}{k} G_{k}-C_{2 \varepsilon} \rho \frac{\varepsilon^{2}}{k}-R_{\varepsilon}
\end{gathered}
$$

\subsection{Combustion model}

The simulation of turbulent reactive flows requests large computational time when the chemical kinetic is involved, [5-6]. The model Probability Density Function (PDF) proposed by Jones \& Whitelaw [7] is an excellent option. A probability function of the mixture fraction provides the temperature and composition values based on tabulated experimental or theoretical data.

$$
\bar{T}=\int_{0}^{1} p(f) T(f) d f
$$

The model solves only two conservation equations: one for the mixture fraction $\mathrm{f}$ and another its variance $\mathrm{f}^{\prime 2}$.

$$
\begin{gathered}
\frac{\partial}{\partial t}(\rho \bar{f})+\frac{\partial}{\partial x_{i}}\left(\rho u_{i} \bar{f}\right)=\frac{\partial}{\partial x_{i}}\left(\frac{\mu_{t}}{\sigma_{t}} \frac{\partial}{\partial x_{i}} \bar{f}\right) \\
\frac{\partial}{\partial t}\left(\rho \overline{f^{\prime 2}}\right)+\frac{\partial}{\partial x_{i}}\left(\rho u_{i} \overline{f^{\prime 2}}\right)=-C_{d} \rho \frac{\varepsilon}{k} \overline{f^{\prime 2}}+ \\
+\frac{\partial}{\partial x_{i}}\left(\frac{\mu_{t}}{\sigma_{t}} \frac{\partial}{\partial x_{i}} \overline{f^{\prime 2}}\right)+C_{g} \mu_{t}\left(\frac{\partial}{\partial x_{i}} \bar{f}\right)^{2}
\end{gathered}
$$

A beta probability function is calculated in each cell based on the mixture fraction, and its variance, eq. (6)

$$
p(f)=\frac{f^{\alpha-1}(1-f)^{\beta-1}}{\int f^{\alpha-1}(1-f)^{\beta-1} d f}
$$

Being the coefficients $\alpha$ and $\beta$ evaluated with equations (7) and (8).

$$
\alpha=\bar{f}\left[\frac{\bar{f}(1-\bar{f})}{\overline{f^{\prime 2}}}-1\right]
$$

$$
\beta=(1-\bar{f})\left[\frac{\bar{f}(1-\bar{f})}{\overline{f^{\prime 2}}}-1\right]
$$


An input of the model is the tabulated results. Figure 3 shows a sample for natural gas.

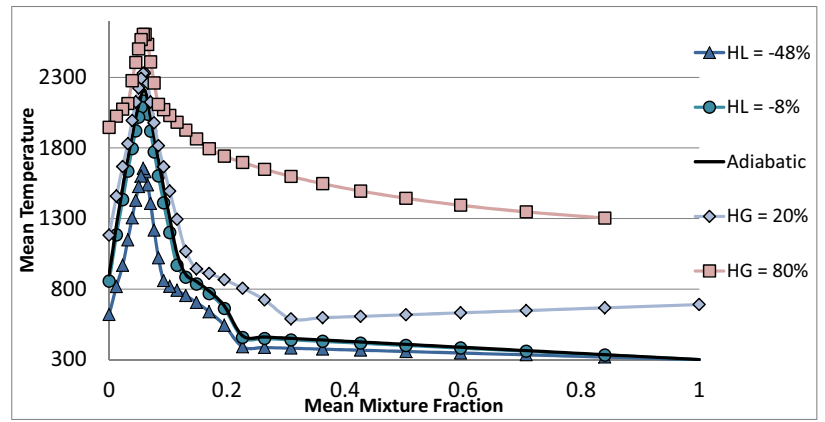

Fig. 3. Tabulated temperature based on mixture fraction and scaled heat loss (HL) and heat gain (HG)

\section{Flow pattern for non-reactive case}

This section intends to stablish the accuracy of the numerical model for non-reactive case. That is the validity of the turbulence model, mesh resolution and numerical algorithm.

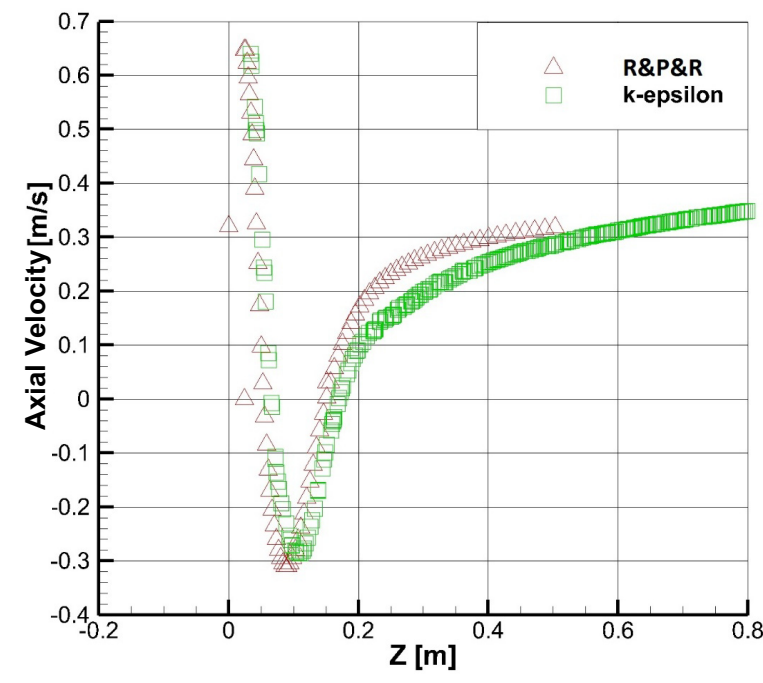

Fig. 4. Validation of the profile of axial velocity along the axis of the chamber. Swirl =1.2. Triangles: experimental data [8], rectangles: numerical results.

Figure 4 shows the longitudinal profile of the axial velocity for swirl number equals to 1.2. The line is the axis of the chamber. Then negative values correspond with the IRZ and are limited by two stagnation points. It is clear that experimental results from [8] and numerical results provide the same IRZ's size. However the numerical results are more dissipative than the experimental ones because they provide a weaker gradient.

\section{Flow pattern for reactive case}

This section is devoted to the study of the reactive cases. Figures 5 depict the radial profiles of axial velocities for Swirl number 1.2 in two sections: at a distance of $25 \mathrm{~mm}$ and $100 \mathrm{~mm}$ from the nozzles' discharge.

At $25 \mathrm{~mm}$ it is possible to identify the momentum transfer between the two jets, the annular and the central one. At $100 \mathrm{~mm}$ the shear layer has suffer a radial displacement because of the IRZ.

As for the comparison of the non-reactive and the reactive cases, the combustion produces an expansion of the reaction's products and then, axial velocities are higher because mass balance must be accomplished.

Recirculation zones are evident in locations with negative axial velocity. Bearing in mind the IRZ, it is evident the combustion's tendency to minimize the IRZ's size.
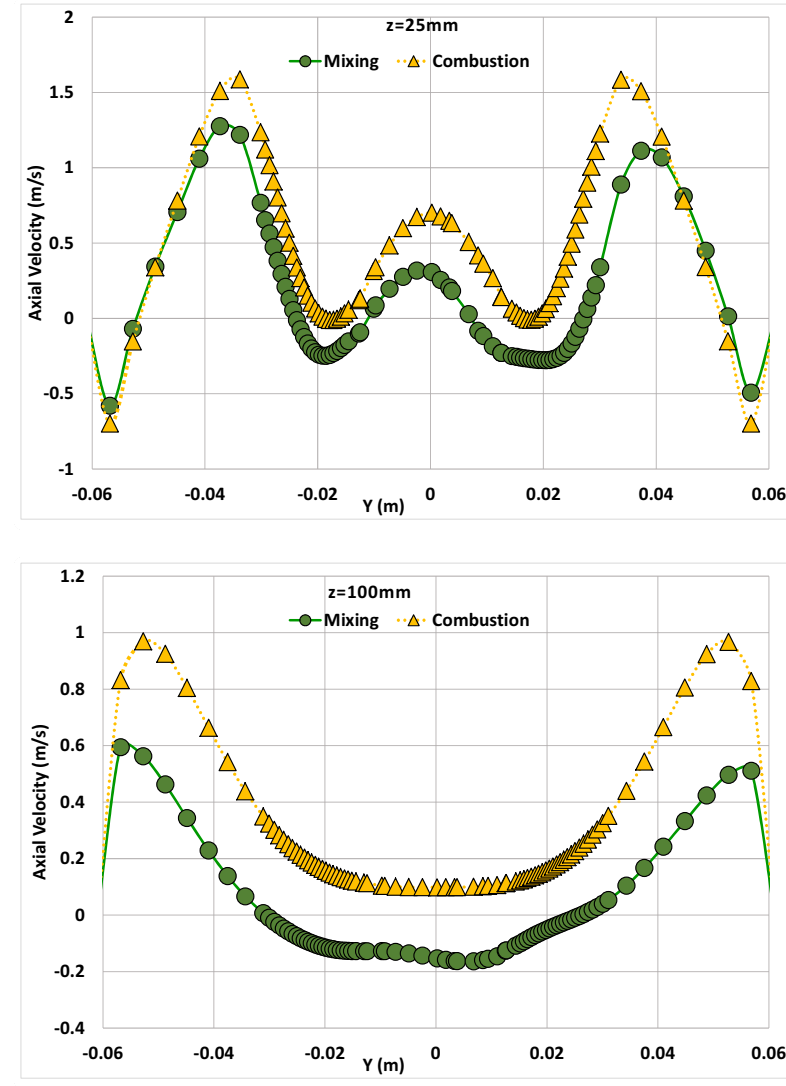

Fig. 5. Radial profile of axial velocity. Swirl $=1.2$. Solid line: non-reactive case. Dash line: reactive case.

Figures 6 show a slice from the end of the swirl generator in the nozzle located in $Z=-50 \mathrm{~mm}$ to $\mathrm{Z}=400 \mathrm{~mm}$. The combustion chamber is $1 \mathrm{~m}$ long to prevent the influence of the outlet in in flow field.

Both figures 6 evidence the mixture is homogenous in a length around 3 diameters of the combustion chamber.

Figure 6 left shows the contours of axial velocity in a longitudinal plane of the chamber. There are two recirculation zones: the annular ring in touch with the walls and the central one near the axis. 
The first one is due to the sudden expansion of the burner whereas the second one is because to the high swirl number of the annular jet. This figure evidence a size of the IRZ for combustion lower than that of figure 4 for non-reactive case.

Figure 6 right shows the contours of temperature in a longitudinal plane. The maximum gradient of temperatures identifies the flame front. This corresponds with the shear layer between the jets, where mixture occurs. The annular ring, near the wall, and the IRZ have high temperature and help to warm the fresh mixture. It is clear the IRZ has the role to fix the location of the flame front and therefore is mainly composed by reaction products. The walls are thermal insulated that is why the lines of iso-temperature cut in perpendicular to the chamber's walls.

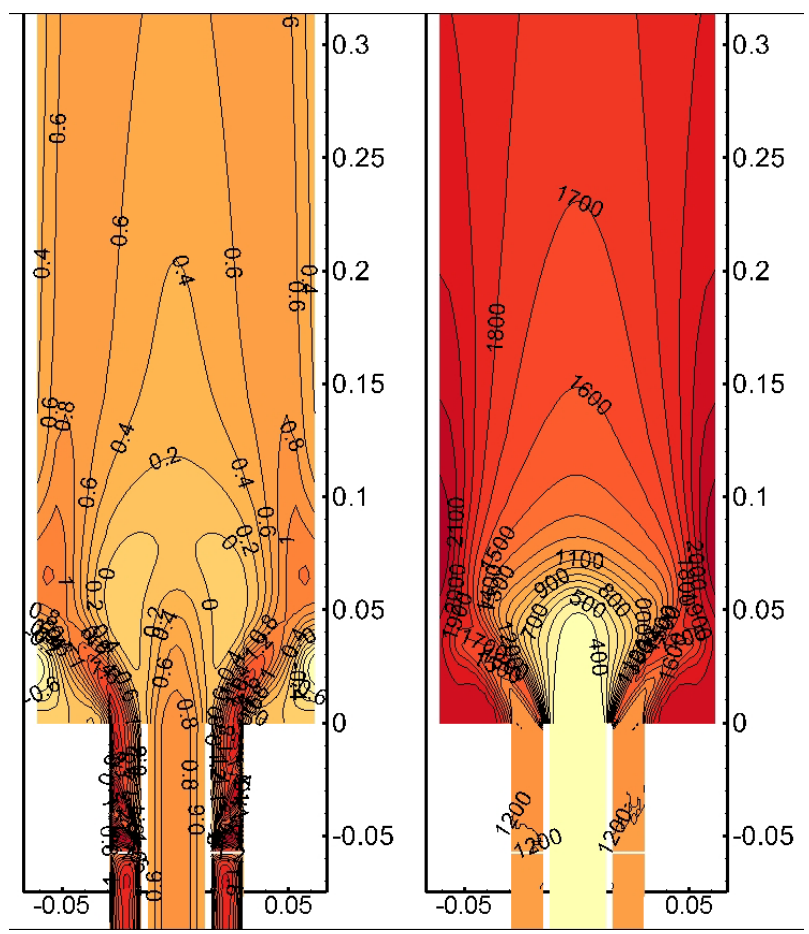

Fig. 6. Longitudinal contour of axial velocity (left) and temperature (right). Swirl $=1.2$.

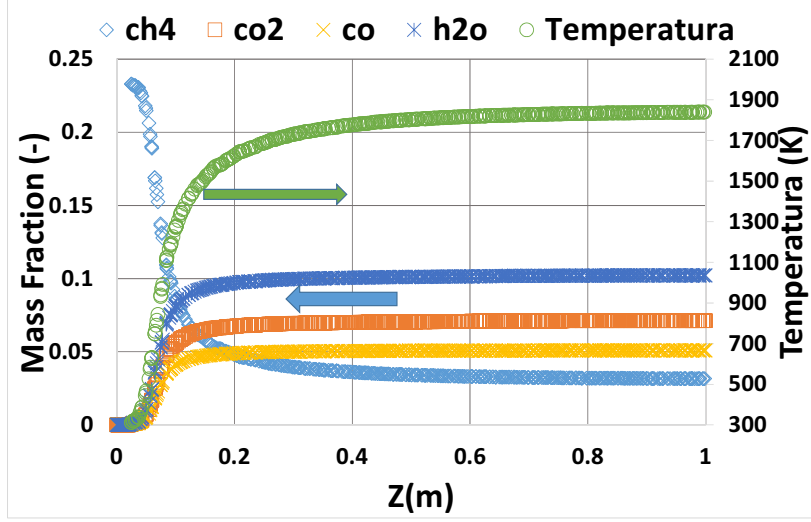

Figure 7. Axial profiles of mass fraction and temperature for Swirl $=1.2$.
Figure 7 depicts the longitudinal profiles of temperature and mass fraction of different components along the axis of the chamber. The profiles are smooth because the local thermodynamic state of the flame provided by the PDF combustion model is weighted based on the beta probability function. Also the turbulence model is RANS; that means that the flow field is averaged in time.

Hence, this PDF model is not suitable for the study of instabilities associated to PVC (Precessing Vortex Core). The main advantage of this model is it requests little computational resources. The chemical kinetics is a stiff problem that needs fine temporal and spatial resolution, [9].

\section{Influence of the diffuser}

This section is devoted to the study of the influence of the diffuser. A conical diffuser of $120^{\circ}$ angle was included between the nozzles' discharge and the test chamber.

Figures 8 show the contours of axial velocity and temperature. The main effect of the diffuser is that prevents the formation of the annular ring recirculation near the walls.

The case with the diffuser produces a larger IRZ. As a result, mixing is better. The mixture is homogeneous in a length around 2 diameters of the chamber.

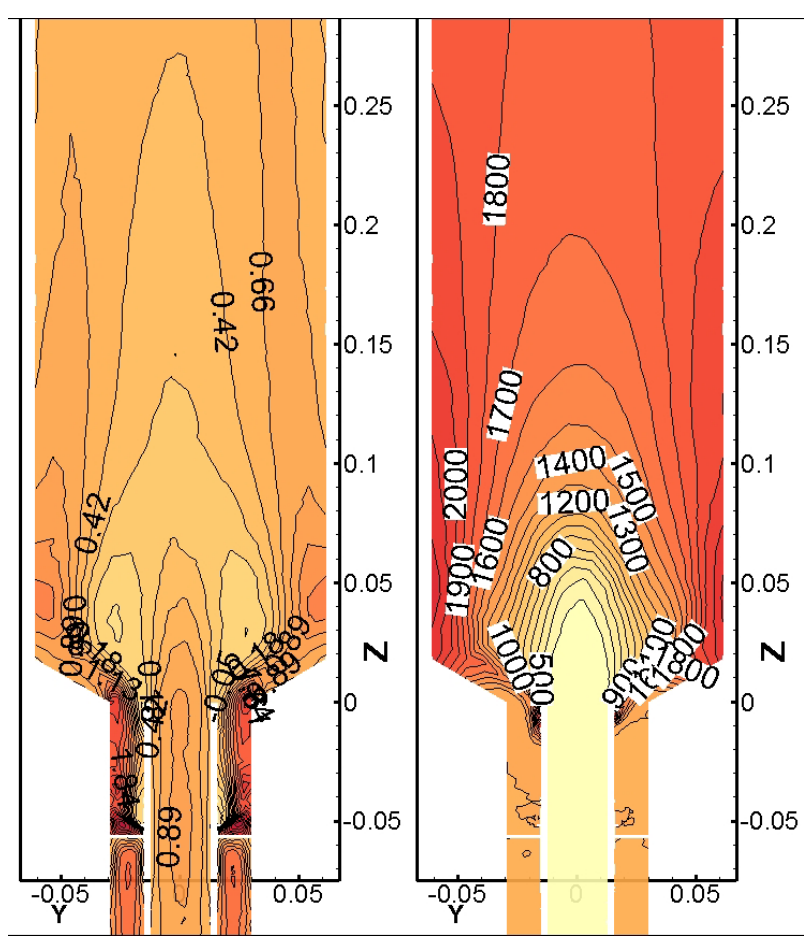

Fig. 8. Longitudinal contour of axial velocity (left) and temperature (right). Swirl $=1.2$. Conical diffuser of $120^{\circ}$

This kind of burner has the problem of high temperatures in the walls because of the confinement of the flame, see figure 6 right and 8 right. This could produce thermal fatigue of the burner. Future works will reduce the degree of confinement of the burner. 
Figures 9 show $\mathrm{f}^{\prime 2}$, the mixture fraction variance, that is a representation of the flame front location in both cases: with and without diffuser.

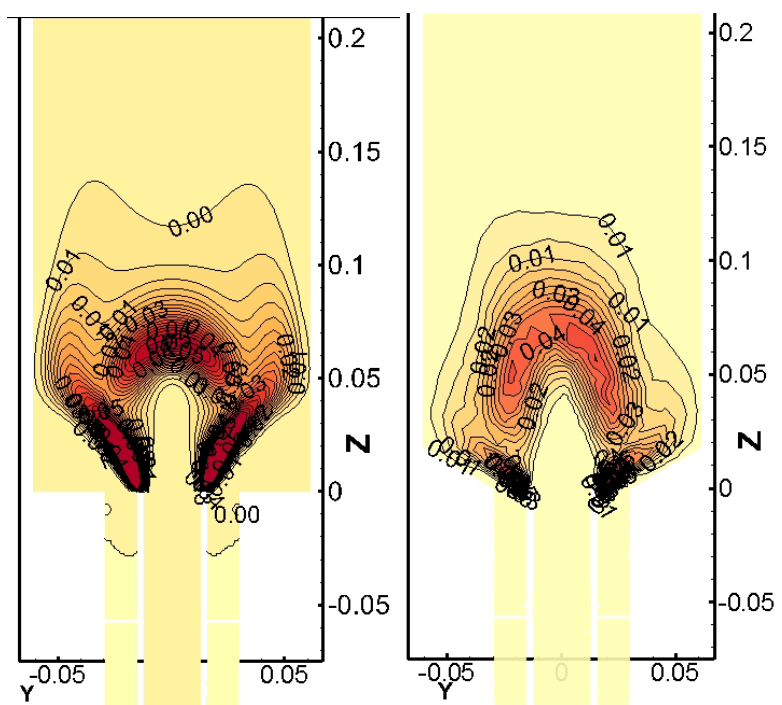

Fig. 9. Contours of mixture fraction variance Swirl $=1.2$. With diffuser (right), without it (left).

\section{Conclusions}

A confined diffusive swirling flame of natural gas has been simulated using CFD.

The set up corresponds with a swirling burner where two coaxial jets are to be mixed. Because of a swirler generator, the vortex breakup appears. This Inner Recirculation Zone is responsible of an enhanced heat and mass transfer. A secondary recirculation zone is the annular ring because of the boundary layer separation.

Combustion is a challenging numerical task because the wide range of characteristic times involved with the chemical kinetics, the convective transport and the diffusive one. The aim was to find a suitable model for reactive turbulent flows with little requisites of computational resources.

The conclusion is that the PDF model based on tabulated experimental o equilibrium results provides tendencies of the flame behaviour with good convergence.

In the case of combustion, IRZ is smaller than the non-reactive case, because of the increase on axial velocities. Besides, both recirculation zones have hot products of reaction that warm the fresh mixture. Also, the IRZ is downwards the flame front and help to keep its location.

Finally, a simulation with a conical diffuser was carried out. The diffuser promotes a larger IRZ, and a shorter mixing.

\section{Acknowledgment}

The author thankfully acknowledges the Spanish Ministry of Science and Innovation for the financial resources in the framework of the project reference ENE2011-25468.

We acknowledge PRACE for awarding us access to resource Curie-GENCI@CEA based in France and MareNostrum@BSC based in Spain. Ref. 2010PA1766

\section{References}

1. R. Roback, B.V. Johnson. NASA CR-168252 (1983)

2. T. Parra-Santos; J. R. Perez-Dominguez; R. Z. Szasz; F. Castro; Engineering Computations, 32/3 (2015) pp. $668-686$

3. T. Parra, J. R. Perez, R. Szasz; et al, EPJ Web of Conferences, 92 (2015) 02059

4. H. K. Versteeg W. Malalasekera. Computational Fluid Dynamics, The finite volume method (1995).

5. K. Kuo. Principles of Combustion. John Wiley and Sons (1986)

6. E. S. Oran and J. P. Boris, Numerical Simulation of Reactive Flow, Elsevier, New York, (1987).

7. W. Jones, J. Whitelaw. Calculation Methods for Reacting Turbulent Flows: A Review. Combustion and Flame 48 (1982) pp. 1-26

8. K. K. J. Ranga Dinesh, M. P. Kirkpatrick, K.W. Jenkins. Investigation of the influence of swirl on a confined coannular swirl jet. Computers \& Fluids 39 (2010) pp. 756-767.

9. J. Warantz, U. Maas and R. W. Dibble. Combustion, Physical and Chemical Fundamentals, Modelling and Simulation, Experiments, Pollutant Formation. editorial Springer Verlag Berlin Heidelberg New York, ISBN 3-540-65228-0, (1999)

10. T. Parra, C. Vega, A. Gallegos; et al, EPJ Web of Conferences, 92 (2015) 02058 